\title{
ON TWISTOR SPACES OF THE CLASS $\mathscr{E}$
}

\author{
F. CAMPANA
}

\section{Introduction}

Let $M^{2 n}$ be a $2 n$-dimensional compact and connected oriented Riemannian manifold, and $Z(M)$ be its twistor space. The $M^{2 n}$ for which $Z(M)$ is Kähler are classified, up to conformal equivalence, in [16], [13] for $n=2$, in [24] for $n \geq 4$ and even, and in [3] for $n \geq 3$. The proofs are mainly differential-geometric.

Y. S. Poon has, however, constructed self-dual metrics on $\mathbb{P}_{2}(\mathbb{C}) \neq$ $\mathbb{P}_{2}(\mathbb{C})=M^{4}$ for which $Z(M)$ is in Fujiki's class $\mathscr{C}$ (i.e., bimeromorphic to a compact Kähler manifold), but not Kähler.

We show here that:

(1) for $n \geq 3, Z(M)$ is in $\mathscr{C}$ iff it is Kähler, iff $M^{2 n}=S^{2 n}$;

(2) for $n=2$, if $Z(M)$ is in $\mathscr{C}$, then $M$ is either $S^{4}$, or homeomorphic to the connected sum of $\tau(M)>0$ copies of $\mathbb{P}_{2}(\mathbb{C})$.

Apart from well-known facts, the proof consists in showing that if $Z(M)$ is in $\mathscr{C}$, then $\pi_{1}(M)=\pi_{1}(Z(M))=0$ where $\pi_{1}$ denotes the fundamental group.

This last equality is obtained by purely complex-geometric methods, using the simple-connectedness of the twistor fibers, and the compactness of the Chow scheme of manifolds in $\mathscr{C}$. More precisely, it is possible (see Theorem 2.2) to evaluate $\pi_{1}(Z)$, for $Z$ in $\mathscr{C}$, from $\pi_{1}(Y)$ and $\pi_{1}(A)$ if $A$ and $Y$ are compact connected submanifolds of $Z$, such that $Y$ has enough "deformations" meeting $A$ in $Z$. When $Y$ is a smooth rational curve with ample normal bundle in $Z$ (for example, a twistor fiber in $\left.Z\left(M^{4}\right)\right)$, and $A$ is a point on $Y$, we get, in particular, $\pi_{1}(Z)=0$. This extends a former result of J. P. Serre on the fundamental group of a unirational variety.

\section{Preliminaries}

1.1 Notation. Let $X$ be any irreducible complex analytic space. Then $\pi_{1}(X):=\pi_{1}(X, a)$ for some unspecified $a$ in $X$.

Received January 5, 1989 and, in revised form, October 24, 1989. 
Let $f: X \rightarrow Y$ be a morphism of irreducible analytic spaces. Then $f_{*}: \pi_{1}(X):=\pi_{1}(X, a) \rightarrow \pi_{1}(Y):=\pi_{1}(Y, f(a))$ denotes the morphism of groups induced by $f$. If no confusion arises, we denote also by $f_{*}$ the morphism induced by the restriction of $f$ to any subspace of $X$.

Let $A$ and $B$ be two irreducible subspaces of $X$, and let $\alpha: A \rightarrow$ $X$ and $\beta: B \rightarrow X$, respectively, be the natural inclusions. Let $\mu$ : $B^{\prime} \rightarrow B$ be any modification of $B$ (for example, its normalization or its desingularization). We shall denote by $\left\langle\pi_{1}(A), \pi_{1}\left(B^{\prime}\right)\right\rangle$ the subgroup of $\pi_{1}(Z)$, generated in $\pi_{1}(Z)$ by $\alpha_{*}\left(\pi_{1}(A)\right)$ and $(\beta \circ \mu)_{*}\left(\pi_{1}\left(B^{\prime}\right)\right)$.

1.2 Remarks. Let $d: X^{\prime \prime} \rightarrow X^{\prime}$ be a desingularization of the normal analytic space $X^{\prime}$. Then $d_{*}$ is surjective, since all fibers of $d$ are connected. However, $d_{*}$ is not always injective: blow-up the vertex of the cone over an elliptic curve.

Let $n: X^{\prime} \rightarrow x$ be the normalization of $X$. Then $n_{*}$ is not always surjective: identify two points in $X^{\prime}=\mathbb{P}_{1}(\mathbb{C})$ to obtain $X$.

1.3 Proposition. Let $f: X \rightarrow Y$ be a proper surjective morphism of irreducible analytic spaces. Assume $Y$ is normal. Then $\left(f_{*} \cdot \pi_{1}(X)\right)$ has finite index in $\pi_{1}(Y)$.

Proof. Let $f:=h \circ g$, where $g: X \rightarrow Y_{0}$ has connected fibers so that $\left(g_{*}\right)$ is surjective, and $h: Y_{0} \rightarrow Y$ is finite surjective. We can thus assume that $f=h$ and $Y_{0}=X$.

Let $Y^{*}$ be a dense Zariski open subset of $Y$ over which $f$ is an unramified covering. Let $X^{*}:=f^{-1}\left(Y^{*}\right)$; then $f_{*}\left(\pi_{1}\left(X^{*}\right)\right)$ has finite index in $\pi_{1}\left(Y^{*}\right)$. The assertion now follows from the following commutative diagram:

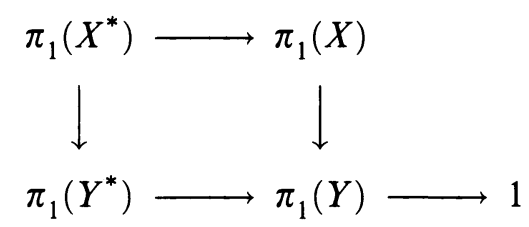

in which the exactness of the bottom line follows from the normality of $Y$, since any $y \in Y$ has a fundamental basis of (contractible) neighborhoods $U$ in $Y$ such that $U^{*}:=\left(U \cap X^{*}\right)$ is pathwise connected.

1.4 Proposition. Let $f: X \rightarrow S$ be a surjective analytic map between irreducible compact analytic spaces, with $S$ normal and $X$ smooth. Let $X_{s}$ be a connected component of a smooth fiber $f^{-1}(s)$ of $f$, and let $Y$ be a compact irreducible analytic subset of $X$ such that $f(Y)=S$. Then $\left\langle\pi_{1}(Y), \pi_{1}\left(X_{s}\right)\right\rangle$ is a subgroup of finite index in $\pi_{1}(X)$.

Proof. Let $S^{*}$ be a dense Zariski open subset of $S$ over which $f$ is smooth. Let $X^{*}$ be $f^{-1}\left(S^{*}\right)$, and let $Y^{*}:=\left(X^{*} \cap Y\right)$. The following 
homotopy sequence provides an exact sequence of groups:

$$
\pi_{1}\left(X_{s}\right) \rightarrow \pi_{1}\left(X^{*}\right) \rightarrow \pi_{1}\left(S^{*}\right) .
$$

(We may assume by Stein reduction, as in Proposition 1.3, that the fibers of $f$ are connected.) Thus, $\left\langle\pi_{1}\left(X_{s}\right), \pi_{1}\left(Y^{*}\right)\right\rangle$ has finite index in $\pi_{1}\left(X^{*}\right)$, and hence in $\pi_{1}(X)$ since $X$ is smooth. Hence $\left\langle\pi_{1}\left(X_{s}\right), \pi_{1}(Y)\right\rangle$ has finite index in $\pi_{1}(X)$, by the functoriality of $\pi_{1}$.

\section{The main result}

2.0 Notation. All analytic spaces here are reduced. Let $Z, A$, and $S$ be compact irreducible analytic spaces, where $A$ is a subspace of $Z$, and $S$ is a subspace of $C(Z)$, the analytic space of compact, pure dimensional, analytic cycles of $Z$ constructed in [2].

Let $G_{s} \subset S \times Z$ be the graph of the universal analytic family $\left(Y_{s}\right)$, $s \in S$, of cycles of $Z$ parametrized by $S$, and let $p: G_{s} \rightarrow S$ and $q: G_{s} \rightarrow Z$ be the restriction of the natural projections of $S \times Z$. Recall that, set-theoretically, $G_{s}:\left\{(s, z)\right.$ s.t. $\left.z \in Y_{s}\right\}$. We call $\left(Y_{s}\right)_{s \in S}$ simply the "family $S$ ". We say that $S$ is $Z$-covering if $q$ is surjective. Equivalently, this means that any $z$ of $Z$ belongs to at least one member of the family $S$. Because $S$ is compact and $Z$ is irreducible, it is sufficient to check this condition for $z$ in some open nonempty subset of $Z$.

Finally, $C(Z)_{A}$ denotes the closed analytic subset of $C(Z)$ consisting of cycles of $Z$ meeting $A$. Thus, $S$ is contained in $C(Z)_{A}$ iff, for any $s$ in $S, Y_{s}$ meets $A$.

2.1 Definition. Let $(Z, A, S)$ be as in Notation 2.0. Then $Z$ is said to be $(A, S)$-connected if:

(1) $Z$ is normal,

(2) $Y_{s}$ is irreducible for $s$ generic in $S$,

(3) $S$ is contained in $C(Z)_{A}$,

(4) $S$ is $Z$-covering.

2.2 Theorem. Let $Z$ be $(A, S)$-connected. Let $s$ be generic in $S$, and $n: Y_{s}^{\prime} \rightarrow Y_{s}$ be the normalization of $Y_{s}$. Then $\left\langle\pi_{1}(A), \pi_{1}\left(Y_{s}^{\prime}\right)\right\rangle$ is of finite index in $\pi_{1}(Z)$.

2.3 Remark. In particular, $\left\langle\pi_{1}(A), \pi_{1}\left(Y_{s}\right)\right\rangle$ and $\left\langle\pi_{1}(A), \pi_{1}\left(Y_{s}^{\prime \prime}\right)\right\rangle$ are of finite index in $\pi_{1}(Z)$ if $d: Y_{s}^{\prime \prime} \rightarrow Y_{s}$ is a desingularization of $Y_{s}$.

2.4 Corollary. Let $Z$ be $(A, S)$-connected. Then the following hold:

(i) If $\pi_{1}(A)=0$ (in particular, if $A=\{a\}$ is a single point of $Z$ ), then $\pi_{1}\left(Y_{s}^{\prime}\right)$ is of finite index in $\pi_{1}(Z)$. 
(ii) If $\pi_{1}\left(Y_{s}^{\prime}\right)=0$, then $\pi_{1}(A)$ is of finite index in $\pi_{1}(Z)$.

(iii) If $\pi_{1}(A)=\pi_{1}\left(Y_{s}^{\prime}\right)=0$, then $\pi_{1}(Z)$ is finite.

Proof of Theorem 2.2. Let $G \subset S^{\prime} \times Z$ be the graph of the family $S^{\prime}$, where $\nu: S^{\prime} \rightarrow S$ is the normalization of $S$. Let $p_{0}: G \rightarrow S^{\prime}$ and $q_{0}: G \rightarrow Z$ be the natural projections. Let $d: G^{\prime} \rightarrow G$ be a desingularization of $G$ and $p^{\prime}:=\left(p_{0} \circ d\right)$ (resp. $q^{\prime}:=\left(q_{0} \circ d\right)$ ). Remark that $G^{\prime}$ is connected. Let $H$ be an irreducible component of $\left(q^{\prime}\right)^{-1}(A)$ such that $p^{\prime}(H)=S^{\prime}$. The existence of $H$ follows from Definition 2.1(3).

By Proposition 1.4, we get that $\left\langle\pi_{1}\left(G_{s}^{\prime}\right), \pi_{1}(H)\right\rangle$ has finite index in $\pi_{1}\left(G^{\prime}\right)$ if $G_{s}^{\prime}:=\left(q^{\prime}\right)^{-1}(s)$ is smooth.

Since $Z$ is normal, $\left(q^{\prime}\right)_{*}\left(\pi_{1}\left(G^{\prime}\right)\right)$ has finite index in $\pi_{1}(Z)$ (Proposition (1.4)). Hence $q_{*}^{\prime}\left(\left\langle\pi_{1}\left(G_{s}^{\prime}\right), \pi_{1}(H)\right\rangle\right)=\left\langle q_{*}^{\prime} \cdot \pi_{1}\left(G_{s}^{\prime}\right), q_{*}^{\prime} \cdot \pi_{1}(H)\right\rangle$ has finite index in $\pi_{1}(Z)$. However, $\left(q_{*}^{\prime} \cdot \pi_{1}\left(G_{s}^{\prime}\right)\right)=\left(\pi_{1}\left(Y_{s}^{\prime}\right)\right)$ in $\pi_{1}(Z)$, and $\left(q_{*}^{\prime} \cdot \pi_{1}(H)\right)$ is contained in $\pi_{1}(A)$. Hence the assertion.

2.5 Remark. Even when $A=(a)$ is a point of $Z$, and $Y_{s}$ is smooth for generic $s$ in $S$, it may happen that $\pi_{1}\left(Y_{s}\right) \neq \pi_{1}(Z)$.

Let, for example, $C$ be a genus 2 curve, let $\alpha^{\prime}: C \rightarrow T^{\prime}$ be its Albanese map, let $\beta: C \rightarrow \mathbb{P}_{3}(\mathbb{C})$ be an embedding, and let $\gamma: T^{\prime} \rightarrow T$ be a degree $d$ isogeny. Also, let $\alpha:=(\gamma \circ d)$, let $f:(\alpha \times \beta): C \rightarrow T \times \mathbb{P}_{3}(\mathbb{C}):=Z$, let $a^{\prime}$ be any point of $C$, and let $a:=f\left(a^{\prime}\right)$. Then $f_{*} \cdot \pi_{1}(C)$ has index $d$ in $\pi_{1}(Z)$, although $Z$ is easily seen to be $(\{\alpha\}, S)$-connected if $S$ is the irreducible component of $C(Z)_{\{\alpha\}}$ containing the point of $C(Z)$ corresponding to $f(C)$.

\section{Rationally connected manifolds}

3.1 Definition. Let $Z$ be a normal irreducible compact analytic space. Then $Z$ is said to be rationally connected, or R.C. for short (resp. smoothly rationally connected, or S.R.C. for short), if there exists $(A, S)$ as in Notation 2.0 such that:

(1) $Z$ is $(A, S)$-connected,

(2) $A=\{\alpha\}$ is a single point of $Z$,

(3) $Y_{s}$ is a rational curve (resp. a smooth rational curve) for $s$ generic in $S$.

3.2 Remarks. (1) It follows from [9, Theorem 3, p. 206, and Remark, p. 208] that $Z$ is Moishezon if $Z$ is rationally connected.

(2) If $f: Z \rightarrow Z^{\prime}$ is surjective (resp. an unramified covering) and $Z$ is R.C. (resp. $Z^{\prime}$ is S.R.C), then $Z^{\prime}$ is R.C. (resp. $Z$ is S.R.C.). In 
particular, taking $Z=\mathbb{P}_{n}(\mathbb{C})$, we see that unirational varieties are R.C., and even S.R.C., if smooth.

(3) $Z$ is R.C. iff $Z_{1}:=Z \times \mathbb{P}_{1}(\mathbb{C})$ is S.R.C., as one sees by considering the graph of the composite map $\mathbb{P}_{1}(\mathbb{C}) \rightarrow Z$ of the normalization of $Y_{s}$, for $s$ generic in $S$, and of the inclusion of $Y_{s}$ in $Z$.

(4) Let $Z$ be smooth and in $\mathscr{C}$. From [17] it follows that $Z$ is S.R.C. (resp. R.C.) iff it contains a smooth rational curve $C$ (resp. a rational curve $C$ ) such that $N Z_{C}$ (resp. $T Z_{\mid C}$ ) is ample, where $N Z_{C}$ (resp. $T Z_{\mid C}$ ) is the normal bundle to $C$ in $Z$ (resp. the restriction to $C$ of the tangent bundle of $Z$ ).

3.3 Question. Let $Z$ be an R. C. manifold. Is it unirational? Probably not, in general. Observe that the answer is obviously negative if $Z$ is not smooth (take the cone over an elliptic curve).

3.4 Proposition. Let $Z$ be an $R$. C. manifold. Then $h^{r}\left(Z, \mathscr{O}_{z}\right)=0$ for $r>0$ where $h^{r}$ is the dimension of the $r$ th-cohomology group $H^{r}\left(Z, \mathscr{O}_{Z}\right)$. In particular, the Euler-Poincaré characteristic $\chi\left(Z, \mathscr{O}_{Z}\right)=1$.

Proof. Since $Z$ is Moishezon, it is sufficient by Hodge symmetry to show that $h^{0}\left(Z, \Omega_{Z}^{r}\right)=0$ for $r>0$. Let $p^{\prime}: G^{\prime} \rightarrow S$ and $q^{\prime}: G^{\prime} \rightarrow Z$ be as in the proof of Theorem 2.2. Let $(s, z)$ be a smooth point of $G_{S^{\prime}}$, with $s$ (resp. $z$ ) smooth in $S$ (resp. $Z$ ), and with $G_{s}^{\prime}:=q^{\prime-1}(s)$ smooth and $q$ of maximal rank of $(s, z)$. Let $\omega \in H^{0}\left(Z, \Omega_{z}^{r}\right)$, let $\Delta$ be any $(r-1)$-dimensional polydisk of $S^{\prime}$ centered at $s$, and let $u$ be any nowhere vanishing section of $\left(\Omega_{\Delta}^{r-1}\right)$. The holomorphic form $\left[\omega_{\Delta} /\left(p^{\prime}\right)^{*} u\right]$ on $G_{s}^{\prime}$ thus vanishes identically, since $G_{s}^{\prime}$ is a rational curve, for any such choice, where $\omega_{\Delta}:=\left(q^{\prime}\right)^{*}(\omega)_{\mid\left(p^{\prime}\right)^{-1}(\Delta)}$. For some neighborhood $U$ of $s$ in $S$, there thus exists a section $v$ of $\left(\Omega_{u}^{r}\right)$ such that $\left(q^{\prime}\right)^{*} \cdot \omega=\left(p^{\prime}\right)^{*} \cdot \nu$. Since $d^{-1}(U \times\{a\})$ is mapped to $a$ by $q^{\prime}, v$ and thus $\omega$ vanish.

3.5 Theorem. Let $Z$ be rationally connected. Then $\pi_{1}(Z)=0$.

Proof. We can assume that $Z$ is S.R.C; possibly we replace it by $Z \times$ $\mathbb{P}_{1}(\mathbb{C})$. Since $\pi_{1}(Z)$ is finite by 2.2 , the universal cover $u: \widetilde{Z} \rightarrow Z$ of $Z$ is S.R.C., so $\tilde{\chi}=\chi\left(\tilde{Z}, \mathscr{O}_{\widetilde{Z}}\right)=1$. On the other hand, $\tilde{\chi}$ is also the degree of the map $u$ by Riemann-Roch.

\section{Moishezon twistor spaces}

4.1 Notation. Let $M=\left(M^{2 n}, g,+\right)$ be a compact connected oriented $2 n$-dimensional $(n \geq 2)$ Riemannian manifold. Let $\tau: Z(M) \rightarrow M$ be its twistor space as constructed in [4] for arbitrary $n$, and in [1], [5, §14], 
[11], [20], [22] for $n=2$. The almost complex structure of $Z(M)$ is integrable precisely when $g$ is self-dual, if $n=2$, and $g$ is conformally flat, if $n \geq 3$. The fibers of $\tau$, called twistor fibers of $Z(M)$, are then rational homogeneous manifolds.

4.2 Proposition. Let $Z_{p}:=\tau^{-1}(p)$ be the reduced twistor fiber of $Z(M)$ above $p \in M^{2 n}$. Let $\left\{Z_{p}\right\}$ be the corresponding point of $C(Z(M))$. Then $C(Z(M))$ is smooth and of dimension $2 n$ at $\left\{Z_{p}\right\}$.

Proof. If $n=2$, this follows from [17], since $Z_{p} \simeq \mathbb{P}_{1}(\mathbb{C})$ has a normal bundle in $Z(M)$ isomorphic to $\mathscr{O}(1)^{\oplus 2}$ [1].

If $n \geq 3$, this follows from [24], since $h^{0}\left(Z_{p}, N\right)=2 n$, where $N$ is the normal bundle of $Z_{p}$ in $Z(M)$, and since $Z_{p}$ has a neighborhood in $Z(M)$ analytically isomorphic to a neighborhood of the zero section in $N$, because $M$ is then conformally flat.

4.3 Definition. Using Proposition 4.2, there exists a unique irreducible component $Z M$ of $C(Z(M))$ containing all $\left\{Z_{p}\right\}$ for $p$ in $M$. The map $t: M^{2 n} \rightarrow Z M$ such that $t(p)=\left\{Z_{p}\right\}$ is then a differentiable totally real embedding of $M^{2 n}$ in the smooth locus of $Z M$. We call $Z M$ the complexification of $M$; it has (complex) dimension $2 n$, but it is not compact in general (see Theorem 4.5 below).

4.4 Proposition. Let $p \in M^{2 n}$, let $A:=Z_{p}$ for $n \geq 3$, and let $A=\{a\}$ with $a \in Z_{p}$ for $n=2$. Let $S$ be the irreducible component of $(Z M)_{A}:=$ $\left(Z M \cap C(Z(M))_{A}\right)$ containing $\left\{Z_{p}\right\}$. Then $Z(M)$ is $(A, S)$-connected iff $S$ is compact.

Proof. By the definition of $(A, S)$-connectedness, we have only to show the "if" part, and so that $S$ is $Z(M)$-covering.

If $n=2$, this follows immediately from [17].

Assume that $n \geq 3$. It is sufficient to show the assertion when $M^{2 n}=$ $S^{2 n}$, since $M$ is then conformally flat. We can thus [24] differentiably identify $N$ with $Z_{p} \times T_{p} M$, where $T_{p} M$ is the tangent space to $M^{2 n}$ at $p$, in such a way that for any holomorphic section $S$ of $N$ over $Z_{p}$, there exists $(u, v) \in\left(T_{p} M\right)^{2}$ such that $s(\tau)=u+\tau \cdot v$, where $Z_{p}$ is identified with the set of complex structures $\tau$ on $T_{p} M$ compatible with both $g$ and $(+)$. Thus $s$ vanishes at $\tau_{0}$ if $v=\tau_{0} u$, and $s$ vanishes somewhere iff $u^{2}=g(u, u)=g(v, v)=v^{2}$ and $u \cdot v=g(u, v)=0$. From this we get that $s(\tau)=w$ iff there exists $h$ which is $g$-orthogonal to $w$ and $\tau w$, and such that $u=w / 2+h$ and $v=w / 2-h$. The conditions $u+\tau v=w$, $u^{2}=v^{2}$, and $u \cdot v=0$ are thus always compatible. Hence the assertion. 
4.5 Theorem. Let $M=\left(M^{2 n}, g,+\right)$ be as in Notation 4.1 and such that the complex structure of $Z(M)$ is integrable. Then the following conditions are equivalent:

(1) $(Z M)$ is compact.

(2) $Z(M)$ is in Fujiki's class $\mathscr{C}$ (i.e., bimeromorphic to some compact Kähler manifold).

(3) $Z(M)$ is Moishezon.

Moreover, in each case, $\pi_{1}(M)=0$.

Proof. The implications $(3) \Rightarrow(2) \Rightarrow(1)$ are generally true (the last one follows basically from [6]; see [14] or [19].)

We show that (1) implies $\pi_{1}(M)=0$. We use the notation of Proposition 4.4. Since $Z(M)$ is $(A, S)$-connected, and $(Z M)$ is compact, $\pi_{1}(A)=0, \pi_{1}\left(Y_{s}\right)=0$ for $s$ generic in $S$, and $\pi_{1}(Z(M) p)=0$ for all $p$ in $M^{2 n}$, it follows from Theorem 2.2 that $\pi_{1}(M)=\pi_{1}(Z(M))$ is finite.

If $n=2, Z(M)$ is then rationally connected, thus Moishezon and with $\pi_{1}(Z(M))=0$. If $n \geq 3, \pi_{1}(M)$ is thus finite.

Let $M^{\prime}$ be the (Riemannian) universal covering of $M$; it is conformally equivalent to $S^{2 n}$ [18]. Then $Z(M)$ is covered by $Z\left(M^{\prime}\right)$ which is rational homogeneous [24], hence rationally connected. Thus $\pi_{1}(Z(M))=0$, and $M$ is conformally equivalent to $S^{2 n}$.

We have thus shown:

4.6 Corollary. Let $M$ be conformally flat. Then the following are equivalent:

(1) $(Z M)$ is compact.

(2) $Z(M)$ is Moishezon.

(3) $Z(M)$ is rational homogeneous (hence projective).

(4) $M$ is conformally equivalent to $S^{2 n}$.

From this we get a purely Riemannian characterization of $S^{4}$, relaxing condition $\pi_{1}\left(M^{4}\right)=0$ in Kuiper's theorem:

4.7 Corollary. Let $M=\left(M^{4}, g,+\right)$ be conformally flat with $b_{1}\left(M^{4}\right)$ $=0$ and $g$ having positive scalar curvature where $b_{1}$ denotes the first Betti number. Then $M$ is conformally equivalent to $S^{4}$.

Proof. From [7] it follows that $b_{2}\left(M^{4}\right)=0$ where $b_{2}$ denotes the second Betti number. Since $b_{1}\left(M^{4}\right)=0$, we get $\chi\left(M^{4}\right)=2$ and $\tau\left(M^{4}\right)=$ 0 . Using [16], $c_{1}^{3}(Z(M))=16\left(2 \chi\left(M^{4}\right)-3 \tau(M)\right)>0$, where $c_{1}$ is the first chern class of the tangent bundle, and $c_{1}^{3}$ its third self-intersection. But Corollary 3.8 of [15] and Serre duality show that $h^{2}\left(Z(M), K_{Z(M)}^{-m}\right)=0$ 
for $m>0$. Riemann-Roch now shows that the Kodaira dimension of $K_{Z(M)}^{-1}$ is 3. Hence $Z(M)$ is Moishezon. The result now follows from Corollary 4.6.

4.8 Remark. Easy examples show that the above conditions do not characterize $S^{m}$ for $m \geq 5$, and that the condition on scalar curvature cannot be removed.

4.9 Corollary. Assume that $M=\left(M^{4}, g,+\right)$ is self-dual and that $Z(M)$ is Moishezon. Then either $M^{4}=S^{4}$ or $M^{4}$ is homeomorphic to the connected sum of $\tau(M)>0$ copies of $\mathbb{P}_{2}(\mathbb{C})$.

Proof. It is sufficient to show that $b_{2}^{-}(M)=0$ [12], [10] since $\pi_{1}(M)=$ 0 . From [16], where $c_{i}=c_{i}(Z(M)), \chi:=\chi(M)$, and $\tau:=\tau(M)$, we have $c_{1} \cdot c_{2}=12(\chi-\tau)$. By Riemann-Roch we have $c_{1} \cdot c_{2}=24$. $\chi\left(Z(M), \mathscr{O}_{Z(M)}\right)=24$, since $Z(M)$ is then rationally connected. Hence $\chi=\tau+2$. On the other hand $b_{1}(M)=0$, so we have $\chi=b_{2}+2$. Hence $b_{2}^{-}(M)=0$, as desired.

4.10 Added in proof. Recently, C. Lebrun and then $H$. Kurke have constructed examples of Moishezon twistor spaces with $M^{4}$ a connected sum of an arbitrary number of copies of $\mathbb{P}_{2}(\mathbb{C})$. As far as the topology of $M^{4}$ is concerned, 4.9 is thus optimal. Question: Does 4.9 remain true with "homeomorphic" replaced by "diffeomorphic"?

\section{Bibliography}

[1] M. Atiyah, N. Hitchin \& I. Singer, Self-duality in four-dimensional Riemannian geometry, Proc. Roy. Soc. London Ser. A 362 (1978) 425-461.

[2] D. Barlet, Familles analytiques de cycles paramétrées par un espace analytique réduit, Lecture Notes in Math., Vol. 482, Springer, Berlin, 1975, 1-158.

[3] P. de Bartolomeis, L. Migliorini \& A. Nanniccini, Espaces de twisteurs Kählériens, C. R. Acad. Sci. Paris Sér. I Math. 307 (1988) 259-261.

[4] L. Berard-Bergery \& T. Ochiai, On some generalizations of the construction of twistor spaces, Global Riemannian geometry (T. J. Willmore and N. J. Hitchin, eds.), Ellis Horwood, 1984.

[5] A. L. Besse, Einstein manifolds, Ergebnisse der Math. 3, Band 10, Springer, Berlin, 1987.

[6] E. Bishop, Conditions for the analyticity of certain sets, Michigan Math. J. 11 (1964) 289-304.

[7] J. P. Bourguignon, Les variétés de dimension 4 á signature non nulle et á courbure harmonique sont d'Einstein, Invent. Math. 63 (1981) 263-286.

[8] F. Campana, Algebricité et compacité dans l'espace des cycles, Math. Ann. 251 (1980) 7-18.

[9] __ Coréduction algébrique d'un espace analytique faiblement Kählérien, Invent. Math. 63 (1981) 187-223.

[10] S. Donaldson, An application of gauge theory to the topology of 4-manifolds, J. Differential Geometry 18 (1983) 269-316. 
[11] M. Dubois-Violette, Structures complexes au-dessus des variétés. Aplications, Séminaire École Norm. Sup., 1981.

[12] M. Freedman, Topology of 4-dimensional manifolds, J. Differential Geometry 17 (1982) $357-454$.

[13] T. Friedriech \& F. Kurke, Compact four-dimensional self-dual Einstein manifolds with positive scalar curvature, Math. Nachr. 106 (1982) 271-299.

[14] A. Fujiki, On automorphism groups of compact Kähler manifolds, Invent. Math. 44 (1978) 225-258.

[15] N. Hitchin, Linear field equations on self-dual spaces, Proc. Roy Soc. London Ser. A 370 (1980) 173-191.

[16] _ Kählerian twistor spaces, Proc. London Math. Soc. 43 (1981) 133-150.

[17] K. Kodaira, $A$ theorem of completeness of characteristic systems for analytic families of compact submanifolds of a complex manifold, Ann. of Math. (2) 75 (1962) 146-162.

[18] N. Kuiper, On conformally flat spaces in the large, Ann. of Math. (2) 50 (1949) 916-924.

[19] D. Lieberman, Compactness of the Chow scheme, Lecture Notes in Math., Vol. 670, Springer, Berlin, 1978, 140-185.

[20] R. Penrose, Nonlinear gravitons and curved twistor theory, General Relativity and Gravitation 7 (1976) 31-52.

[21] Y. S. Poon, Compact self-dual manifolds with positive scalar curvature, J. Differential Geometry 24 (1986) 97-132.

[22] S. Salamon, Quaternionic Kähler manifolds, Invent. Math. 67 (1982) 143-171.

[23] J. P. Serre, On the fundamental group of a unirational variety, J. London Math. Soc. 34 (1959) 481-484.

[24] M. Slupinski, Espaces de twisteurs Kähleriens en dimension $4 k, k>1$, Thèse, École Polytechnique, Massy-Palaiseau,1984.

UNIVERSITÉ NANCY

FRANCE 
